\title{
Performance Testing in Architectural Design: Evolving the Problem-Solving Paradigm for Novice Designers
}

\author{
Paola Sanguinetti \\ University of Kansas, USA \\ paolas@ku.edu
}

\begin{abstract}
This paper compares two approaches to introduce novice architectural designers to computational tools for performance testing. Focus is placed on parametric design changes to test solutions. The first approach is limited to the design of a shading component. The second approach consists of short exercises using Building Information Modeling to test and analyze discrete decisions. The hurdles in the learning process are discussed, including the role of visualization to evaluate performance. The effectiveness on learning strategies is discussed.
\end{abstract}

Keywords: Design education; BIM; Performance-based design; Novice designer; Design problem-solving

\section{Introduction}

Computational tools can potentially help optimize the architectural design process, by providing performance feedback to the exploration of a design solution and supporting the ability to embed knowledge in the model. These tools also present a big learning curve for novice designers. One important issue is that computational tools to aid designers involve a variety of modeling representations, including the use of parametric modeling to explore options based on project criteria and dynamic simulation modeling for environmental performance evaluation. Performance-based design is a key concept that permeates in the integration of these new tools for design support. The results of two courses are compared, where performance testing is presented as an approach to learn through problem-solving and evaluating environmental performance in architecture education. The effectiveness of learning strategies is discussed in terms of the comprehension of building performance.

\section{Performance-based design}

The Performance Based Building framework is a validation mechanism which quantifies and evaluates the building's performance against a target performance (Foliente, Leicester, \& Pham, 1998). In architectural practice, Building Information Modeling (BIM) provides a technological platform to support performance-based design. The process involves the use of various computational tools to examine design solutions against performance criteria. Many advances have been made to improve interoperability and data exchanges between computational tools for design and performance evaluation (C. M. Eastman, 1992; C. Eastman, Jeong, Sacks, \& Kaner, 2009). A model has been proposed to automate the analysis of building models in early concept design (Sanguinetti et al., 2012). However it has also been pointed out that the application of performance-based design in early design stages is difficult because of the lack of integration with form generation or modification needed as part of the architectural design problem-solving process (Oxman, 2008). A recent study has found ha the architecture problem is very "broad" which impedes the formalization knowledge toward a performance-based design approach (Fischer, 2006).

\section{Design-based learning}

Architectural design activities have been described ill-defined problem-solving due to the open-ended nature of both the problem and its solution (C. M. Eastman, 1969; Simon, 1974). The problem-solving paradigm has been adopted in education as a way to engage learning through tackling complex problems that can be solved in multiple ways (Hmelo-Silver, 2004). In this context, a difference has been established between skill and competency, where skill refers to the level of knowledge, and competency, describes the ability to produce a valuable solution to a problem (Chyung, Stepich, \& Cox, 2006). Research in the process of design has found that the framing the problem is the first step in the process followed by an iterative exploration of the solution (Cross, 2004). In design education, iteration in the design process is an active way for students to acquire knowledge through exploration and testing of ideas (Gómez Puente, Van Eijck, \& Jochems, 2011; Jolley, 2013). The speed and ease in solving the design problem is associated to the knowledge and experience of the designer (Kavakli \& Gero, 2002).

\section{Performance Testing}

The methodology proposed in this paper, focuses on the activities of novice designers to acquire knowledge about the performance of a system using of computational tools (Jonassen \& Hung, 2006). Performance Testing is referred to as the iterative diagnostic 
process integrating environmental analysis tools to visualize the performance of a design solution. Two approaches for performance testing are presented where novice designers are introduced to computational tools for design and analysis iteration. The first approach is limited to a façade design problem where solutions are tested and analyzed parametrically. The second approach consists of short exercises to introduce to analyze decisions based on parametric design changes.

\section{Canopy design configuration for daylight control}

In the approach, the design and environmental analysis tools are integrated as plugin to a Rhino, a NURBS-based geometric modeling tool. Introductory tutorials for grasshopper parametric modeling concepts included: a) parametric relationships and constraints, b) conditional statements, c) design rules. Students learn to develop parametric components, and build the model as a bottom-up assembly. The second set of tutorials was organized for analysis using Diva, a plugin interface for daylight analysis. Performance testing is used to evaluate the shading system parameters to control the folding of the surface:

- Translation (folded surface point location)

- Multiplier (number of components)

- $\quad$ Scale (size of the component)

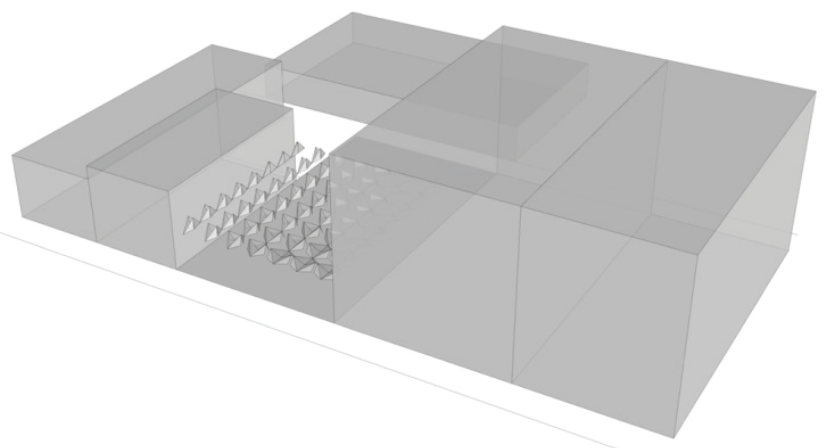

Figure 1: Geometric model produced in Rhino and parametric canopy modeled in Grasshopper.
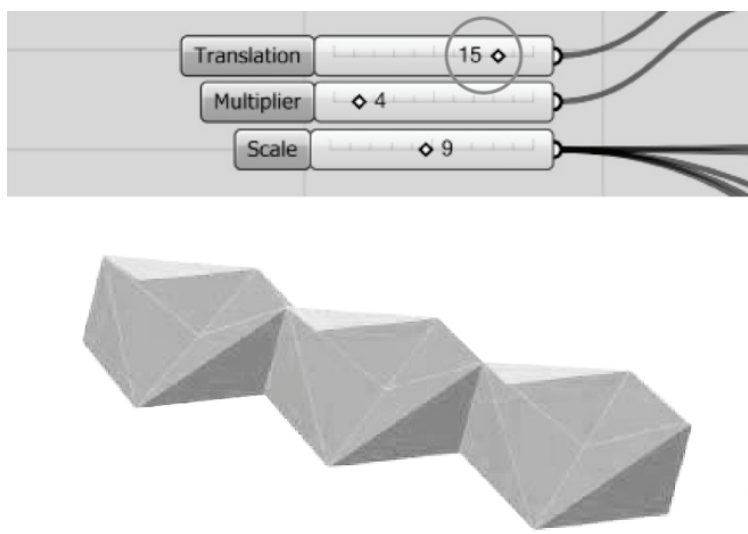

Figure 2: Parametric component for the canopy.

\section{Window configuration for natural ventilation and shading design for daylight control}

In the approach, introductory tutorials of BIM concepts included: a) geometric representation, b) spatial and object relationships, c) properties and quantities of building components, d) geographic information. Concepts of building science and performance in the areas of materials, structures, environmental systems are introduced as lectures. Four performance aspects for sustainable design were explored though the use of building information modeling: the configuration of shading devices for daylight control; the configuration of window openings to promote natural ventilation; the selection and layout of structural members to reduce material use; and the selection of materials and paneling configuration to improve the acoustic by reducing reverberation. This paper presents the exercise for daylight performance and natural ventilation.

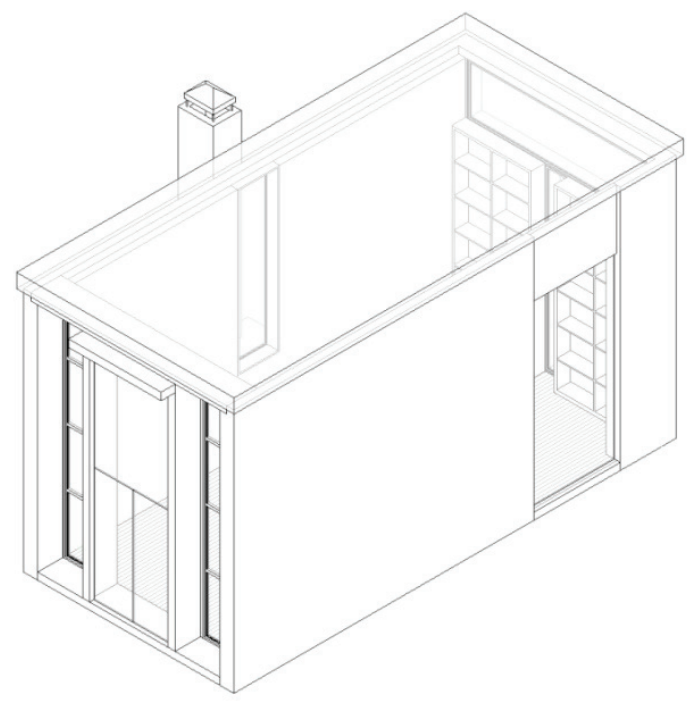

Figure 3: Geometric base model produced in Revit

For the natural ventilation performance analysis, the building model is exported to a simulation tool to visualize airflow through building openings at different times of the year and in different latitudes. The assumption is that the openings refer to operable windows or doors in the building. Performance testing to control airflow and promote natural ventilation is based on the size and position of openings. Performance testing is used to evaluate the window parameters:

- Opening height

- Opening width

- $\quad$ Ratio of wall surface to window opening area

Performance criteria for the shading system involved the control of direct light sunlight on the east window opening. For the south elevation the goal was to maximize winter solar gain and minimize summer solar gain. In addition, the shading device configuration should maximize visual connection from inside to outside. 
Performance testing is used to evaluate the shading system parameters:

- Louver depth

- Louver angle

- $\quad$ Louver spacing

\section{Results}

The testing process consists of changing design parameters and visualizing and evaluating the effects of these parametric variations. Figure 4 shows the results of performance testing using the first approach, where the geometric parameters of a canopy design are manipulated and the resulting solar irradiation analysis is mapped as a color gradient on the ground surface of the model.

Figure 5 and 6 show the results of performance testing in the second approach. Performance evaluation involves the documentation of the changes of the design parameters under different environmental conditions including changes in geographical locations and temporal conditions. Figure 5 shows how Wind velocity and air pressure are documented for three different locations, at two times of the year: winter (January) and summer (August); and at three times of the day (8:00 am, 12:00 noon, 4:00 pm). Figure 6 shows the varying shading conditions for the spring equinox, the summer and winter solstice in Oslo, Norway; Havana, Cuba, and Lawrence, Kansas, USA.

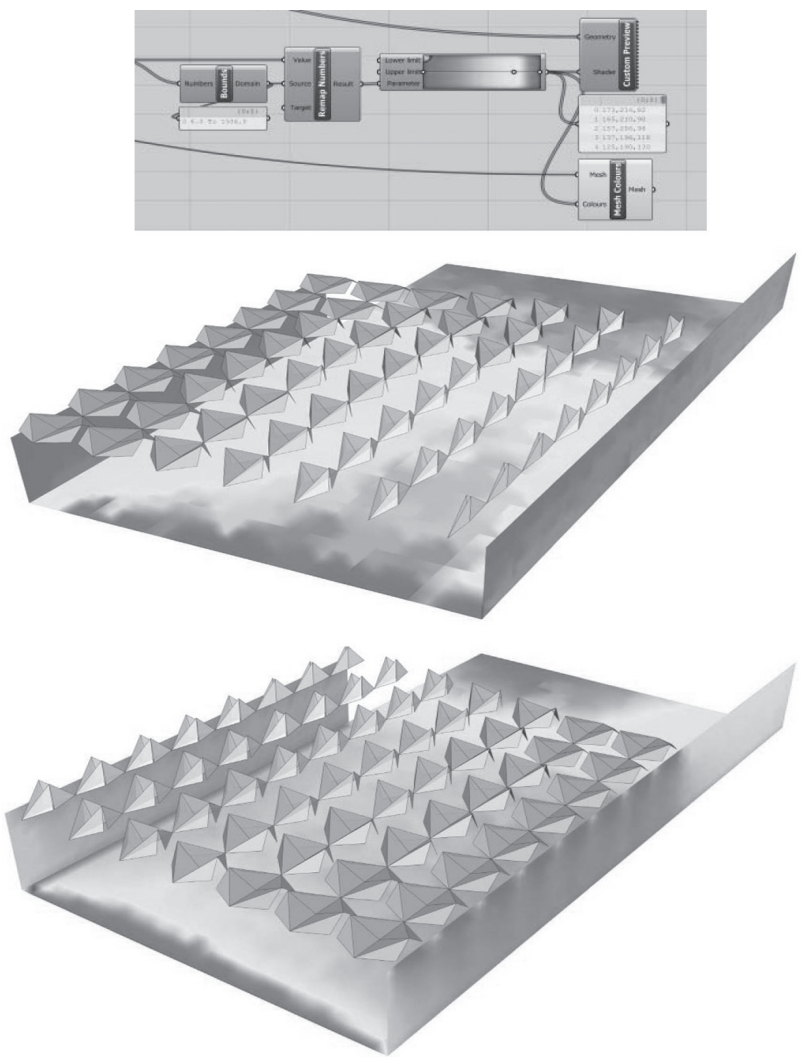

Figure 4: Example of a parametric definition in grasshopper with two model iterations showing the color gradient representing solar irradiation values under the canopy.
Lawrence, Kansas: Scenario 1

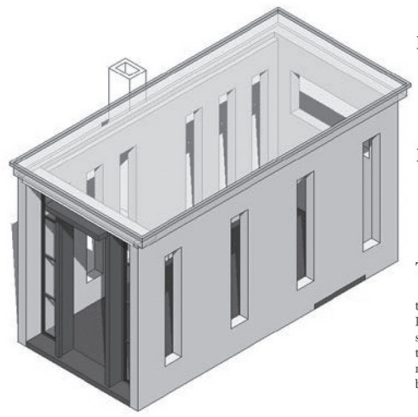

Parameters (East/West Facades) Opening Sill Height- 3 Opening Height- $116^{\prime}$
Opening Width- $2^{\prime}$ Ratio of Wall to Opening Area- 46/217 Number of Windows- 10 Parameters (North/South Facades) Opening Sill Height- $0 ; 10^{\circ}$

Opening Height- $16^{6} 6^{\prime}, 4^{\prime} 6^{\prime \prime}$

Ratio of Wall to Opening Number of Windows- 2 Total Ratio of Wall to Opening Area- 509/1085 Lawrence Kansas has relatively strong to medium winds during the summer and winter that come $\mathrm{SW}$ and $\mathrm{SSW}$ so for the first scenario
L wanted to test the effect of having openings on all sides of the bust see what that would do to the air flow. I expected to have more air moving through the structure and less spots were the air was calm to stagnant. M
main reason for this is because the summers are very hot so it would be
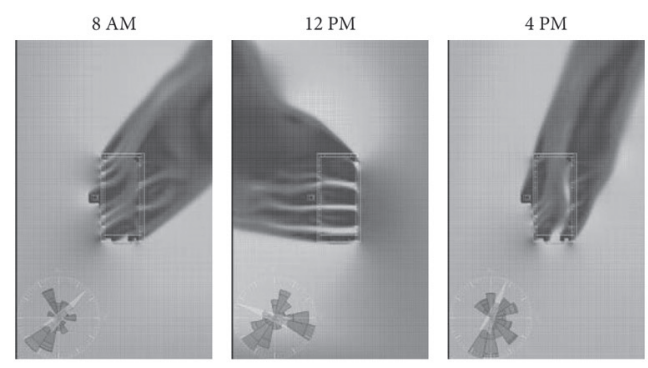

Summer
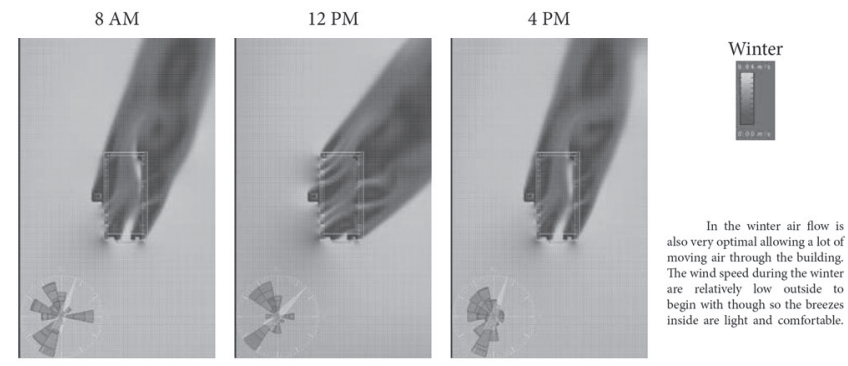

Figure 5: Example of student documentation of performance testing for natural ventilation.

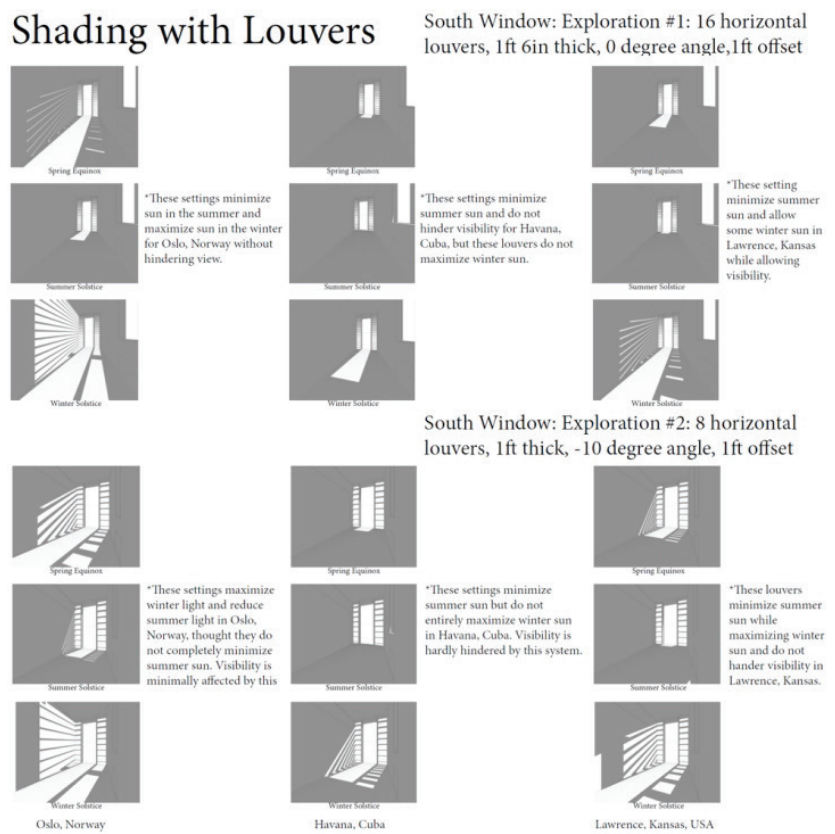

Figure 6: Example of student documentation of solar exposure under varying environmental conditions and latitudes. 


\section{Conclusion}

This study focuses on the activities of novice architectural designers using commercial software tools for analysis and visualization for design problem-solving. Performance testing is proposed as an evolution of the problem-solving paradigm where students diagnose the proposed solution by evaluating its performance and "trouble shooting". Performance testing supports an effective approach to formalize and integrate analysis into the design process. Two design-based learning approaches are presented where the iterative design process is constrained through the use parametric modeling. Both approaches integrate technical and domain knowledge in discrete design exercises where students explore design solutions. The two approaches emphasize the development of conceptual thinking and problemsolving skills, through design exploration and visual demonstration of performance information. The comparison of two approaches for performance testing shows that reducing the solution space proves more successful in enabling novice designers to generate and test design solutions. The success of these discrete exercises is rooted in the visualization comprehension of the expected performance.

\section{References}

Chyung, Seung Youn, Stepich, Donald, \& Cox, David. (2006). Building a competency-based curriculum architecture to educate 21st-century business practitioners. Journal of Education for Business, 81(6), 307314.

Cross, Nigel. (2004). Expertise in design: an overview. Design studies, 25(5), 427-441.

Eastman, Charles M. (1969). Cognitive processes and ill-defined problems: A case study from design. Paper presented at the Proceedings of the International Joint Conference on Artificial Intelligence: IJCAI.
Eastman, Charles M. (1992). Modeling of buildings: evolution and concepts. Automation in Construction, 1(2), 99-109.

Eastman, CM, Jeong, Y-S, Sacks, R, \& Kaner, I. (2009). Exchange model and exchange object concepts for implementation of national BIM standards. Journal of Computing in Civil Engineering, 24(1), 25-34.

Fischer, Martin. (2006). Formalizing construction knowledge for concurrent performance-based design Intelligent Computing in Engineering and Architecture (pp. 186-205): Springer.

Foliente, GC, Leicester, RH, \& Pham, L. (1998). Development of the CIB proactive program on performance based building codes and standards. BCE Doc, 98, 232.

Gómez Puente, SM, Van Eijck, M, \& Jochems, W. (2011). Towards characterising design-based learning in engineering education: a review of the literature. European Journal of Engineering Education, 36(2), 137-149.

Hmelo-Silver, Cindy E. (2004). Problem-based learning: What and how do students learn? Educational Psychology Review, 16(3), 235-266.

Jolley, Victoria. (2013). Constructing Knowledge: a Pedagogical evaluation of Design-based Learning. UCLan Journal of Pedagogic Research, 4.

Jonassen, David H, \& Hung, Woei. (2006). Learning to troubleshoot: A new theory-based design architecture. Educational Psychology Review, 18(1), 77-114.

Kavakli, Manolya, \& Gero, John S. (2002). The structure of concurrent cognitive actions: a case study on novice and expert designers. Design Studies, 23(1), 25-40.

Oxman, Rivka. (2008). Performance-based design: current practices and research issues. International journal of architectural computing, 6(1), 1-17.

Sanguinetti, Paola, Abdelmohsen, Sherif, Lee, JaeMin, Lee, JinKook, Sheward, Hugo, \& Eastman, Chuck. (2012). General system architecture for BIM: An integrated approach for design and analysis. Advanced Engineering Informatics, 26(2), 317-333.

Simon, Herbert A. (1974). The structure of ill structured problems. Artificial intelligence, $4(3), 181-201$. 